INPLASY

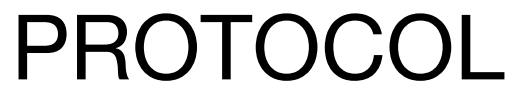

To cite: Liu et al. Risk factors

for multiple primary

melanomas. Inplasy protocol

202180119. doi:

10.37766/inplasy2021.8.0119

Received: 31 August 2021

Published: 31 August 2021

Corresponding author:

Ruolin Liu

912270620@qq.com

Author Affiliation:

Sichuan university

Support: None.

Review Stage at time of this submission: The review has not yet started.

Conflicts of interest:

None declared.

\section{Risk factors for multiple primary melanomas}

$$
\text { Liu, R1; Wan, Q2; Zhao, R³. }
$$

Review question / Objective: To identify the patients at high risk of developing multiple primary melanomas.

Condition being studied: Melanoma.

Information sources: PubMed, Web of Science, Medline, Embase, Cochrane Library.

Main outcome(s): The risk estimates and $95 \% \mathrm{Cl}$. The outcome is defined in the original articles and the pooled relative risks and $95 \% \mathrm{Cl}$ is calculated using Stata.

INPLASY registration number: This protocol was registered with the International Platform of Registered Systematic Review and Meta-Analysis Protocols (INPLASY) on 31 August 2021 and was last updated on 31 August 2021 (registration number INPLASY202180119).

Participant or population: Patients with multiple primary melanoma.

Intervention: Reporting the risk estimates and $95 \%$ confidence interval $[\mathrm{Cl}]$ for risk factors of MPM by comparing with those with single melanoma.

Comparator: Patients with single primary melanoma.

Study designs to be included: Prospective or retrospective cohort studies and casecontrol studies. 
Eligibility criteria: (1) reporting at least one clinical risk factor for MPM patients using univariate or multivariate analysis, (2) reporting the risk estimates and $95 \%$ confidence interval $[\mathrm{Cl}]$ for risk factors of MPM by comparing with those with single primary melanoma.

Information sources: PubMed, Web of Science, Medline, Embase, Cochrane Library.

Main outcome(s): The risk estimates and $95 \% \mathrm{Cl}$. The outcome is defined in the original articles and the pooled relative risks and $95 \% \mathrm{Cl}$ is calculated using Stata.

Quality assessment / Risk of bias analysis: Begg's and Egger's tests will be done to assess the publication bias. The Newcastle-Ottawa Quality Assessment Scale will be used to evaluate study selection, matching, and outcome of the included studies.

Strategy of data synthesis: XAll statistical analyses are going to use Stata statistical software. $Q$ test is going to be used to assess heterogeneity in outcomes across studies, and 12 statistic is used to quantify it. An 12 score of $50 \%$ or higher is considered to show significant heterogeneity. In anticipation of clinical heterogeneity, the random-effects model is performed, otherwise the fixed-effects is performed.

Subgroup analysis: Subgroup metaanalyses were performed in which the subgroup items were analyzed in at least two studies, including the level of education, hair color, Breslow depth, histology, and body site of the initial melanoma.

Sensitivity analysis: None.

Country(ies) involved: China.

Keywords: Melanoma; multiple primaries; risk factors; sun exposure.

Contributions of each author:

Author 1 - Ruolin Liu.
Author 2 - Qianyi Wan. Author 3 - Rui Zhao. 